\title{
Generalized Iteration Method for First-Kind Integral Equations
}

\author{
By Dionisios Margetis and Jaehyuk Choi
}

\begin{abstract}
An iteration method is described to solve one-dimensional, first-kind integral equations with finite integration limits and difference kernel, $K\left(x-x^{\prime}\right)$, that decays exponentially. The method relies on deriving via the Wiener-Hopf factorization and solving by suitable iterations in the Fourier complex plane a pair of integral relations, where each iteration accounts for all end point singularities in $x$ of the exact solution. For even and odd kernels, this pair reduces to decoupled, 2nd-kind Fredholm equations, and the iteration yields Neumann series subject to known convergence criteria. This formulation is applied to a classic problem of steady advection-diffusion in the two-dimensional (2D) potential flow of concentrated fluid. The remarkable overlap of recently derived asymptotic expansions for the flux in this case is shown to be intimately related to the analyticity of the kernel Fourier transform.
\end{abstract}

\section{Introduction}

A class of mixed boundary-value problems (BVPs) in two space dimensions (2D) with Dirichlet conditions on a finite strip can be cast via Green's function [1] to the first-kind integral equation [2-4]

$$
\int_{a}^{b} d x^{\prime} K\left(x-x^{\prime}\right) u\left(x^{\prime}\right)=f(x), \quad a<x<b,
$$

\footnotetext{
Address for correspondence: Dionisios Margetis, 2-380, Department of Mathematics, Massachusetts Institute of Technology, 77 Massachusetts Avenue, Cambridge, MA 02139; e-mail: dio@math.mit.edu 
where the difference kernel, $K(x)$, is integrable in $(a-b, b-a)$ and may be singular at $x=0$, and the source function, $f(x)$, is continuous in $(a, b)$. In particular, (1) with exponentially decaying $K(x)$ as $|x| \rightarrow \infty$, , where $x$ is extended to $(-\infty, a-b) \cup(b-a,+\infty)$, arises in physical situations such as continuous growth by advection-diffusion [5-7], and solidification and freezing from a flowing melt [8-12].

There is no general theory for solving (1) explicitly [13, 14]. Closed-form solutions can be obtained when $a$ and $b$ are finite for certain algebraic and logarithmic kernels [15], and when $a=-\infty$ or $b=+\infty$ for admissible $K(x)$ and $f(x)$ by use of the Fourier transform $[2,3]$. Numerical methods for (1) include Latta's method [16], which relies on the ordinary differential equation (ODE) satisfied by $K(x)$, improvements over Latta's method by conversion of (1) for scattering problems to ODEs satisfied by Painlevé transcendents [17], series expansions in appropriate eigenfunctions [18], and the collocation method [10, 19].

Noteworthy are approximation methods $[3,14]$ in the $x$ - (physical) or Fourier space. One method [3, 20] involves conversion of (1) with oscillatory, even kernel $K(x)$ to two Fredholm-type integral equations of the 2 nd kind in the Fourier domain. The resulting equations are solved approximately via a specialized asymptotic technique [20], which is based on local expansions in the complex plane, appropriate only for calculating diffracted (far) fields in scattering problems. Another method $[13,14]$ is based on splitting the integral in (1) and solving successively Wiener-Hopf equations of the first kind [2]. Both of these methods [3, 14] are applicable to kernels $K(x)$ that are logarithmically singular at $x=0$.

Recently [7] an iterative, fast convergent series in the spirit of Refs. [13, 14] was invented to solve (1) when $\pi e^{-x} K(x)=K_{0}(|x|)$, the modified Bessel function of the third kind [21], $f(x)$ is a constant, and $b=-a=P$, where $P$ is sufficiently large. The Green function for the corresponding BVP obeys an advection-diffusion partial differential equation in $2 \mathrm{D}$ and decays exponentially with distance. In this case (1) describes, for example, the flux normal to the boundary of a finite absorber in the steady 2D potential flow of concentrated fluid [5-7] where $P$ is the Péclet number, which measures the relative importance of advection compared to diffusion. A remarkable finding in [7] is the significant overlap of leading order, disparate expansions for sufficiently "high" and "low" $P$, mainly due to the rapid convergence of the former expansion even for small values of $P, P \geq O\left(10^{-2}\right)$. This success of asymptotic analysis has been puzzling until now.

\footnotetext{
${ }^{1}$ A function $g(x)$ defined in $(-\infty,+\infty)$ is exponentially decaying if $g(x)=O\left(|x|^{-\alpha^{ \pm}} e^{p^{ \pm} x}\right)$ as $x \rightarrow$ $\pm \infty$ where $p^{-}>0, p^{+}<0$ and $\alpha^{ \pm}$are real. For our purposes, this definition includes the case where either $p^{+}$or $p^{-}$equals $\delta$ with $\delta \rightarrow 0$; see Equations (21) and (57) below.
} 
In this article, we formulate a theory with the following features: (i) Approximate treatment of (1) for the sufficiently broad class of kernels $K(x)$ that have algebraic Fourier transforms, relaxing requirements for the behavior of these kernels at $x=0$ yet requiring their exponential decay as $|x| \rightarrow$ $\infty$; and (ii) explanation of the remarkable properties of past, high-Péclet number expansions for solutions to advection-diffusion problems [6, 7]. Our formulation unifies the methods of $[3,14]$ and places the treatment of (1) on the firm grounds of the theory for 2nd-kind Fredholm equations when the Fourier transform of $K(x)$ is defined to be analytic and invertible in a finite strip of the Fourier domain.

Our starting point is the formulation of [3] for oscillatory kernels, which we review here for completeness and extend to other kernels to solve (1) systematically. Equation (1) is converted via the Wiener-Hopf factorization $[2,3,22]$ to a pair of coupled integral relations in the Fourier domain, and then to decoupled, 2nd-kind Fredholm equations by imposition of kernel symmetry. We propose a general, iterative treatment of the coupled equation pair by applying regular perturbation in the Fourier domain; the iterations are enabled by a coupling constant of this pair that decreases exponentially with the physical strip length, $(b-a)$. For odd- and even-symmetric kernels, our iterations yield convergent Neumann series $[2,23]$ if the complex kernel, $\mathcal{K}\left(k, k^{\prime}\right)$, of the corresponding Fredholm equations has finite, appropriate integral measure (norm), to be defined in Section 2.3.2. The iteration method preserves the desirable feature of the methods in $[13,14]$ of yielding at each iteration all singularities of the unknown function at the strip end points, $x=a$ and $b$.

We validate our method by studying the integral equation for the steady advection-diffusion problem studied in [7]. We derive the high- $P$ expansion of [7] by our new iterative means, thus justifying the method invented heuristically in [7], and analyze the convergence of this expansion by relating a lower bound of $P$ required for convergence to analytic properties of the complex kernel, $\mathcal{K}$. Hence, our approach reveals an intimate connection of the iteration convergence for (1) with the analyticity of the kernel Fourier transform.

The article is organized as follows. In Section 2.1, we convert (1) to a pair of coupled integral relations satisfied by suitable Fourier transforms. In Section 2.2, we describe a systematic iteration procedure to solve this equation pair, transcending limitations of approximations made in [3]. In Section 2.3, we focus on odd and even kernels and reduce the equation pair to 2nd-kind Fredholm equations in the complex domain; we discuss conditions for existence and uniqueness of solution, and solve these equations via suitable Neumann series. In Section 3, we illustrate our method for $K(x)=(1 / \pi) e^{x} K_{0}(|x|)$ and $f(x)=1$, studied via different methods in [7-12]: In Section 3.1, we obtain a "high-Péclet" series expansion for the solution of (1); in Section 3.2, we provide the analytical reason for the remarkable convergence of this expansion for small Péclet numbers; and in Section 3.3, we compare the iteration series 
with the exact, numerically evaluated solution of the corresponding BVP. In Section 4, we conclude our analysis with a summary and discussion.

\section{Wiener-Hopf technique and iteration method}

In this section, we start with the application of the Wiener-Hopf method of factorization [2] to (1), assuming that a unique integrable solution, $u(x)$, exists; in Section 2.3, we discuss conditions sufficient for uniqueness by imposing kernel symmetry. We start by following [3] to derive a pair of integral relations for suitably defined Fourier transforms related to $u(x)$. Next, we transcend [3] describing procedures of solving these equations iteratively to arbitrary order under general considerations.

First, we give a few definitions and clarify notation. The kernel $K(x)$, defined for $x \in(-\infty,+\infty)$, has Fourier transform

$$
\tilde{K}(k)=\int_{-\infty}^{\infty} d x K(x) e^{-i k x} .
$$

Similarly, the Fourier transform, $H(k)$, of any other admissible function $h(x)$ defined in $(-\infty,+\infty)$ stems from replacing $K(x)$ by $h(x)$ in the right side of (2). We assume that $\tilde{K}(k)$ is an algebraic function, analytic and invertible in the finite complex strip $\{k: \alpha<\operatorname{Im} k<\beta\}$ where $\alpha<0$ and $\beta \geq 0$. Consistent with this strip of analyticity and (2), two asymptotic limits of $K(x)$ are (see footnote 1)

$$
K(x)=O\left(e^{-\beta x}\right) \quad x \rightarrow+\infty, \quad K(x)=O\left(e^{-\alpha x}\right) \quad x \rightarrow-\infty
$$

see Section 2.2.1 for further details. Throughout this article, the + subscript denotes functions (+ functions) analytic in the "upper half plane," $\{k: \operatorname{Im} k>$ $\alpha\}$, and the - subscript denotes functions (- functions) analytic in the "lower half plane," $\{k: \operatorname{Im} k<\beta\}$.

\subsection{Integral relations in Fourier domain}

This section focuses on the derivation of the following proposition.

Proposition 1 [3] [Reduction of (1) to pair of integral relations]. If $\tilde{K}(k)$ is invertible and analytic inside the strip $\{k: \alpha<\operatorname{Im} k<\beta, \alpha<0, \beta \geq 0\}$, (1) reduces to the coupled integral relations

$$
\begin{gathered}
V_{+}(k)+\frac{H_{+}(k)}{K_{+}(k)}+\int_{C_{1}} \frac{d k^{\prime}}{2 \pi i} \frac{e^{-i k^{\prime}(b-a)} H_{-}\left(k^{\prime}\right)}{K_{+}\left(k^{\prime}\right)} \frac{1}{k^{\prime}-k}=0, \\
C_{1}=\left\{k^{\prime}: \operatorname{Im} k^{\prime}=c_{1}, \alpha<c_{1}<\operatorname{Im} k\right\},
\end{gathered}
$$




$$
\begin{gathered}
S_{-}(k)+\frac{H_{-}(k)}{K_{-}(k)}-\int_{C_{2}} \frac{d k^{\prime}}{2 \pi i} \frac{e^{i k^{\prime}(b-a)} H_{+}\left(k^{\prime}\right)}{K_{-}\left(k^{\prime}\right)} \frac{1}{k^{\prime}-k}=0, \\
C_{2}=\left\{k^{\prime}: \operatorname{Im} k^{\prime}=c_{2}, \operatorname{Im} k<c_{2}<\beta\right\},
\end{gathered}
$$

where $H_{ \pm}(k)$ are the (unknown) Fourier transforms of the functions $\underset{\mathrm{r}}{h_{1}}(x)$ defined by

$$
\begin{aligned}
& h_{1}(x)=\int_{a}^{b} d x^{\prime} K\left(x-x^{\prime}\right) u\left(x^{\prime}\right) \quad x<a, \quad h_{1}(x)=0 \quad x>a, \\
& h_{\mathrm{r}}(x)=\int_{a}^{b} d x^{\prime} K\left(x-x^{\prime}\right) u\left(x^{\prime}\right) \quad x>b, \quad h_{\mathrm{r}}(x)=0 \quad x<b,
\end{aligned}
$$

$K_{ \pm}(k)$ are defined to be free of zeros in the upper (+) and lower (-) half planes with

$$
K_{ \pm}(k)=\exp \left\{ \pm \int_{C_{\frac{1}{2}}} \frac{d k^{\prime}}{2 \pi i} \frac{\ln \tilde{K}\left(k^{\prime}\right)}{k^{\prime}-k}\right\}
$$

$V_{+}(k)$ and $S_{-}(k)$ are related to the source function $f(x)$ by

$$
V_{+}(k)=\int_{C_{1}} \frac{d k^{\prime}}{2 \pi i} \frac{e^{i k^{\prime} a} F\left(k^{\prime}\right)}{K_{+}\left(k^{\prime}\right)} \frac{1}{k^{\prime}-k}, \quad S_{-}(k)=-\int_{C_{2}} \frac{d k^{\prime}}{2 \pi i} \frac{e^{i k^{\prime} b} F\left(k^{\prime}\right)}{K_{-}\left(k^{\prime}\right)} \frac{1}{k^{\prime}-k},
$$

and $F(k)$ is the Fourier transform of $f(x)$ by setting $f(x)=0$ outside $[a, b]$,

$$
F(k)=\int_{a}^{b} d x f(x) e^{-i k x}
$$

Proof: First, we routinely set $u(x)=0$ and $f(x)=0$ for $x<a$ and $x>b$ in (1) and thus obtain the equivalent relation

$$
\int_{-\infty}^{+\infty} d x^{\prime} K\left(x-x^{\prime}\right) u\left(x^{\prime}\right)=f(x)+h_{1}(x)+h_{\mathrm{r}}(x), \quad-\infty<x<\infty,
$$

where $h_{1}$ and $h_{\mathrm{r}}$ are defined by (5). Application of the Fourier transform to (9) yields

$$
\tilde{K}(k) U(k)=F(k)+e^{-i k a} H_{+}(k)+e^{-i k b} H_{-}(k),
$$

where

$$
\begin{aligned}
U(k) & =\int_{a}^{b} d x u(x) e^{-i k x}, \\
H_{+}(k) & =\int_{-\infty}^{0} d x h_{1}(x+a) e^{-i k x}, \quad H_{-}(k)=\int_{0}^{+\infty} d x h_{\mathrm{r}}(x+b) e^{-i k x},
\end{aligned}
$$


and $F(k)$ is given by (8). Because $h_{1}(x)=O\left(e^{-\alpha x}\right)$ as $x \rightarrow-\infty$ and $h_{\mathrm{r}}(x)=O\left(e^{-\beta x}\right)$ as $x \rightarrow+\infty$ in (5), $H_{+}(k)$ is indeed analytic for $\operatorname{Im} k>\alpha$ and $H_{-}(k)$ is analytic for $\operatorname{Im} k<\beta$.

Second, we simplify (10) by formally factorizing $\tilde{K}(k)$,

$$
\tilde{K}(k)=K_{+}(k) K_{-}(k),
$$

where $K_{ \pm}(k)$ are found through the Cauchy integral formula [2, 22] and are described by (6); we define $K_{ \pm}(k)$ to be free of zeros in their half planes of analyticity. Thus, (10) reads

$$
K_{-}(k)\left[e^{i k a} U(k)\right]_{-}=\frac{\left[e^{i k a} F(k)\right]_{-}}{K_{+}(k)}+\frac{H_{+}(k)}{K_{+}(k)}+\frac{\left[e^{-i k(b-a)} H_{-}(k)\right]_{-}}{K_{+}(k)},
$$

or, alternatively,

$$
K_{+}(k)\left[e^{i k b} U(k)\right]_{+}=\frac{\left[e^{i k b} F(k)\right]_{+}}{K_{-}(k)}+\frac{H_{-}(k)}{K_{-}(k)}+\frac{\left[e^{i k(b-a)} H_{+}(k)\right]_{+}}{K_{-}(k)} .
$$

Third, we find a relation between $H_{+}$and $H_{-}$via further decomposing the right-hand sides of (14) into + and - functions,

$$
\begin{aligned}
\frac{\left[e^{-i k(b-a)} H_{-}(k)\right]_{-}}{K_{+}(k)} & =W_{+}(k)+W_{-}(k), \\
\frac{\left[e^{i k(b-a)} H_{+}(k)\right]_{+}}{K_{-}(k)} & =R_{+}(k)+R_{-}(k), \\
\frac{\left[e^{i k a} F(k)\right]_{-}}{K_{+}(k)} & =V_{+}(k)+V_{-}(k), \\
\frac{\left[e^{i k b} F(k)\right]_{-}}{K_{-}(k)} & =S_{+}(k)+S_{-}(k) .
\end{aligned}
$$

Furthermore, $H_{ \pm} / K_{ \pm}$in (14) is analytic in the upper (+, upper sign) and lower (-, lower sign) half plane, since by (6) none of $K_{ \pm}(k)$ vanishes in their respective half plane of analyticity. Thus, by means of analytic continuation we reduce (14) to the relations [2]

$$
V_{+}+\frac{H_{+}}{K_{+}}+W_{+}=0, \quad S_{-}+\frac{H_{-}}{K_{-}}+R_{-}=0,
$$

where each term in (19) is assumed to vanish as $|k| \rightarrow \infty$ in the upper (left equation) or lower (right equation) half plane. Relations (4), along with (7), follow from (19) by expressing $W_{+}, R_{-}, V_{+}$, and $S_{-}$as contour integrals 
of the left sides in (15), (16), (17), and (18) using the Cauchy integral formula $[2,22]$. This statement concludes the proof.

The reader is referred to Section 2.2.1 for details on the singularities ascribed to $\tilde{K}(k)$. We now state the following corollary.

COROLlaRY 1. Once $H_{ \pm}(k)$ are determined by solving (4), $u(x)$ is obtained via the inversion formula

$u(x)=\int_{\Gamma} \frac{d k}{2 \pi} U(k) e^{i k x}=\int_{\Gamma} \frac{d k}{2 \pi} \frac{F(k)+e^{-i k a} H_{+}(k)+e^{-i k b} H_{-}(k)}{\tilde{K}(k)} e^{i k x}$,

where $\Gamma$ is the infinite, parallel to the real axis, path that lies in the strip $\{k: \alpha<$ $\operatorname{Im} k<\beta\}$, provided that the integral (20) converges.

\subsection{Iteration method for kernel $K(x)$ without odd or even symmetry}

In this section, we prescribe more precisely the kernels entering (1) and apply an iteration method to solve (4) by treating the integrals involving $H_{ \pm}$as small in some sense.

2.2.1. Prescriptions for kernel Fourier transform. We introduce the following interrelated prescriptions.

(i) $\tilde{K}(k)$ is analytic and invertible in the strip $\left\{k:-2 \xi_{0}<\operatorname{Im} k<0\right\}$. Consistent with this complex strip,

$K(x)=O\left(x^{-\eta}\right) \quad x \rightarrow+\infty, \quad K(x)=O\left(|x|^{-\zeta} e^{2 \xi_{0} x}\right) \quad x \rightarrow-\infty$,

where $\eta$ and $\zeta$ are positive for all practical purposes.

(ii) $K_{-}(k)$ has poles or branch points at $k=k_{p}^{\text {up }}$ (in the upper half plane) where $p=0,1, \ldots, \operatorname{Im} k_{p+1}^{\text {up }} \geq \operatorname{Im} k_{p}^{\text {up }}$, and $\operatorname{Im} k_{0}^{\text {up }} \equiv 0$. The requisite branch cuts lie in the upper half plane. By $(21), \tilde{K}(k)=O\left(\left(k-k_{0}^{\text {up }}\right)^{\eta-1}\right)$ as $k \rightarrow k_{0}^{\text {up }}$.

(iii) $K_{+}(k)$ has poles or branch points at $k=k_{p}^{\text {lo }}$ (in the lower half plane) where $\operatorname{Im} k_{p+1}^{\mathrm{lo}} \leq \operatorname{Im} k_{p}^{\mathrm{lo}}$ and $\operatorname{Im} k_{0}^{\mathrm{lo}}=-2 \xi_{0}<0$. The requisite branch cuts lie in the lower half plane. ${ }^{2}$ By $(21), \tilde{K}(k)=O\left(\left(k-k_{0}^{\text {lo }}\right)^{\zeta-1}\right)$.

By our prescriptions, the kernel $K(x)$ is not odd nor even, although odd and even kernels may be suitably transformed to meet (i)-(iii) (see footnote 2). Evidently, $\beta=0$ and, thus, the lower half plane is the region $\{k: \operatorname{Im} k<0\}$, and $\alpha=-2 \xi_{0}$ and the upper half plane is the region $\left\{k: \operatorname{Im} k>-2 \xi_{0}\right\}$.

\footnotetext{
${ }^{2}$ The prescribed locations of singularities may be obtained for any kernel having $\tilde{K}(k)$ analytic and invertible in a strip of the $k$-plane with finite width, $2 \xi_{0}$. It suffices to multiply $K(x), u(x)$, and $f(x)$ in (1) by suitable, simple exponential functions. Hence, kernels such as $K(x)=e^{A x} H_{0}(|x|)$, where $H_{0}(z)$ is the modified Hankel function [21], are excluded from our analysis.
} 
2.2.2. Iterations in Fourier domain. To indicate that (4) are amenable to iterations, we next indicate that these equations have a natural coupling constant when $\tilde{K}(k)$ is analytic and invertible in a finite strip. Further justification of iterations is given in Section 2.3.2 by invoking an appropriate kernel norm within the theory for 2nd-kind Fredholm equations. With the change of the integration variable by $k^{\prime} \Rightarrow k^{\prime}-i 2 \xi_{0}$ in the integral of (4a), (4) read

$$
\begin{aligned}
V_{+}(k)+\frac{H_{+}(k)}{K_{+}(k)}+\epsilon \int_{C_{+}} \frac{d k^{\prime}}{2 \pi i} \frac{e^{-i k^{\prime}(b-a)} H_{-}\left(k^{\prime}-i 2 \xi_{0}\right)}{K_{+}\left(k^{\prime}-i 2 \xi_{0}\right)} \frac{1}{k^{\prime}-i 2 \xi_{0}-k}=0, \\
S_{-}(k)+\frac{H_{-}(k)}{K_{-}(k)}-\int_{C_{-}} \frac{d k^{\prime}}{2 \pi i} \frac{e^{i k^{\prime}(b-a)} H_{+}\left(k^{\prime}\right)}{K_{-}\left(k^{\prime}\right)} \frac{1}{k^{\prime}-k}=0,
\end{aligned}
$$

where $C_{+}=\left\{k^{\prime}: \operatorname{Im} k^{\prime}=c_{+}=\right.$const.; $\left.0<c_{+}<\operatorname{Im} k+2 \xi_{0}\right\}, C_{-}=C_{2}$ and

$$
\epsilon=e^{-2 \xi_{0}(b-a)}, \quad \epsilon<1 \text {. }
$$

For algebraic kernels that satisfy criteria (i)-(iii) of Section 2.2.1, we deform each integration path in (22) in the lower, for (22a), or upper, for (22b), half $k^{\prime}$-plane by wrapping the contour around branch cuts and evaluating residues at poles. Evidently, the integrals are $O(1)$ as $\epsilon \rightarrow 0$. So, the solution to (22) is now formulated as a problem of regular perturbation.

The main proposition of this section is stated as follows.

PROPOSITION 2 [Regular perturbation and dominant-balance equations for (4)]. Replacing $H_{ \pm}(k)=H_{ \pm}(k ; \epsilon), V_{+}=V_{+}(k ; \epsilon)$, and $S_{-}=S_{-}(k ; \epsilon)$ in (22) by the power-series expansions

$$
\begin{gathered}
H_{ \pm} \sim \sum_{n=0}^{N} \epsilon^{n} H_{n \pm}, \quad H_{n \pm}=O(1) \\
V_{+} \sim \sum_{n=0}^{N_{1}} \epsilon^{n} V_{n+}, \quad S_{-} \sim \sum_{n=0}^{N_{2}} \epsilon^{n} S_{n-}, \quad V_{n+}, S_{n-}=O(1) \quad \epsilon \rightarrow 0^{+},
\end{gathered}
$$

and taking $K_{ \pm}(k)$ as independent of $\epsilon, H_{n \pm}$ are determined recursively by

$$
\begin{aligned}
& H_{n+}(k)=-K_{+}(k)\left[V_{n+}(k)+\frac{1}{2 \pi i} \int_{C_{+}} d k^{\prime^{\prime}} \frac{e^{-i k^{\prime}(b-a)} H_{(n-1)-}\left(k^{\prime}-i 2 \xi_{0}\right)}{K_{+}\left(k^{\prime}-i 2 \xi_{0}\right)}\right. \\
&\left.\times \frac{1}{k^{\prime}-i 2 \xi_{0}-k}\right], \quad H_{(-1)-} \equiv 0 \\
& H_{n-}(k)=-K_{-}(k)\left[S_{n-}(k)-\frac{1}{2 \pi i} \int_{C_{-}} d k^{\prime} \frac{e^{i k^{\prime}(b-a)} H_{n+}\left(k^{\prime}\right)}{K_{-}\left(k^{\prime}\right)} \frac{1}{k^{\prime}-k}\right] \\
& n=0,1,2, \ldots
\end{aligned}
$$


Proof: The proof follows after some algebra by direct substitution of (24) and (25) in (22), setting equal coefficients of same powers of $\epsilon$. The details are left as an exercise.

A few remarks on Proposition 2 are in order. (i) Proposition 2 does not address the existence and uniqueness of solution to (4), nor does it provide conditions for the validity of regular perturbation and its possible convergence to a solution; these matters are discussed in Section 2.3 for even and odd kernels, which arise in most physical situations. (ii) The convergence of the iteration series relies both on the magnitude of $\epsilon$ and on properties of the kernels entering (4); the reasons become clear in Section 2.3 via the theory of 2nd-kind Fredholm equations. (iii) $V_{n+}, S_{n-}$ and corresponding integrals in (26) may depend on $a, b$, or $\xi_{0}$, thus implicitly depending on $\epsilon$. The present perturbation approach views $\epsilon$ as a parameter properly independent of $a, b$, or $\xi_{0}$. (iv) In view of the initialization condition in (26a),

$$
H_{0+}(k)=-K_{+}(k) V_{0+}(k)
$$

the zeroth-order iteration, $n=0$, yields a right-end point correction to the problem of the semi-infinite strip. More generally, because approximations for both $H_{ \pm}(k)$ are included at each iteration step $(n=0,1,2, \ldots)$, (26) yield both end point singularities of $u(x)$ at every iteration, $n$; see the example of Section 3.1.

In view of Corollary 1, we state the following.

Corollary 2. Once $H_{n \pm}$ are determined, $U=U(k ; \epsilon)$ and $u=u(x ; \epsilon)$ follow from

$$
U(k ; \epsilon) \sim U_{0}(k)+\sum_{n=1}^{N} \epsilon^{n} U_{n}(k), \quad u(x ; \epsilon) \sim u_{0}(x)+\sum_{n=1}^{N} \epsilon^{n} u_{n}(x),
$$

where, if $F(k)$ is independent of $\epsilon$,

$$
\begin{aligned}
U_{0}(k) & =\frac{F(k)+e^{-i k a} H_{0+}(k)+e^{-i k b} H_{0-}(k)}{\tilde{K}(k)}, \\
U_{n \geq 1}(k) & =\frac{e^{-i k a} H_{n+}(k)+e^{-i k b} H_{n-}(k)}{\tilde{K}(k)}, \\
u_{n}(x) & =\int_{\Gamma} \frac{d k}{2 \pi} U_{n}(k) e^{i k x}
\end{aligned}
$$

and $\Gamma$ lies in $\left\{k:-2 \xi_{0}<\operatorname{Im} k<0\right\}$, provided that the integrals (28d) converge. 


\subsection{Even or odd kernel: Conversion to and solution of Fredholm equations}

In this section, we formulate and solve simplified integral equations for the $H_{ \pm}(k)$ introduced in (4) of Proposition 1 via imposing kernel symmetry. We extend the formulation of [3] to odd kernels and point out connections to 2nd-kind Fredholm equations, which were unnoticed in $[3,13,14]$.

First, we take $b=-a>0$ and impose $\forall x \in(-\infty,+\infty)$

$$
K(-x)=K(x) \text { (even symmetry), or } K(-x)=-K(x) \text { (odd symmetry). }
$$

Applying kernel symmetrization consistent with prescriptions (i)-(iii) of Section 2.2.1, $\tilde{K}(k)$ is analytic and invertible inside the symmetric strip $D=\left\{k:-\xi_{0}=\alpha<\operatorname{Im} k<\beta=\xi_{0}\right\}$ with either $\tilde{K}(-k)=\tilde{K}(k)$ or $\tilde{K}(-k)=-\tilde{K}(k) \forall k \in D$, where $\pm \theta_{0} \pm i \xi_{0}\left(\theta_{0}\right.$ : real) are singularities of $\tilde{K}(k)$ closest to the real axis. Without loss of generality, we take

$$
\begin{aligned}
& K_{+}(-k)=i K_{-}(k) \quad \forall k \in D_{1}=\left\{k: \operatorname{Im} k<\xi_{0}\right\}, \\
& K_{-}(-k)=\mp i K_{+}(k) \quad \forall k \in D_{\mathrm{u}}=\left\{k: \operatorname{Im} k>-\xi_{0}\right\},
\end{aligned}
$$

where the upper or lower sign holds for an even or odd kernel, respectively, and $D=D_{\mathrm{u}} \cap D_{1}$.

Second, we note in particular that, for $u(x)$ and kernels that satisfy the conditions of the Hilbert-Schmidt theorem [23], (1) has a unique solution if the kernel eigenfunctions form a complete set [2, 23]. A sufficient condition for completeness is that $K(x)$ stems from the Green function of a proper Sturm-Liouville system $[2,23]$. This observation applies to square integrable $u(x)$ and $f(x)$, and is thus restricted to its applicability: for example, it cannot account for solutions $u(x)=\left(b^{2}-x^{2}\right)^{-1 / 2} w(x)$, where $w(x)$ is continuous in $[-b, b]$, which stem from kernels $K(x)$ logarithmically singular at $x=0$ [13]. In Section 2.3.2, we discuss the solvability of (1) on the basis of 2nd-kind Fredholm equations in the Fourier domain.

2.3.1. Conversion to 2nd-kind Fredholm equations. Without further ado, we state the following proposition.

PROPOSITION 3 [3] [Formulation of (1) in terms of 2nd-kind Fredholm equations]. For even kernel $K(x), K(-x)=K(x) \forall x \in(-\infty,+\infty)$, (4) are recast to the decoupled Fredholm equations

$$
Y_{-}^{ \pm}(k) \pm \int_{\Gamma^{\mathrm{e}}} d k^{\prime} \mathcal{K}^{\mathrm{e}}\left(k, k^{\prime}\right) Y_{-}^{ \pm}\left(k^{\prime}\right)=\mathcal{S}_{-}^{\mathrm{e}}(k)
$$


where $k$ is real, the integration path $\Gamma^{\mathrm{e}}$ lies in the real axis and is indented below $-k$, the unknown (-) functions are defined $b y^{3}$

$$
Y_{-}^{ \pm}(k)=H_{+}(-k) \pm H_{-}(k),
$$

the source (-) function is

$$
\mathcal{S}_{-}^{\mathrm{e}}(k)=\left[-i V_{+}(-k) \mp S_{-}(k)\right] K_{-}(k),
$$

and the complex kernel $\mathcal{K}^{\mathrm{e}}$ is defined by

$$
\mathcal{K}^{\mathrm{e}}\left(k, k^{\prime}\right)=\frac{1}{2 \pi} \frac{e^{-2 i k^{\prime} b}}{k^{\prime}+k} \frac{K_{-}(k)}{K_{+}\left(k^{\prime}\right)} .
$$

For odd kernel $K(x), K(-x)=-K(x) \forall x \in(-\infty,+\infty)$, (4) are recast to

$$
Z_{+}^{ \pm}(k) \mp \int_{\Gamma^{\circ}} d k^{\prime} \mathcal{K}^{\mathrm{o}}\left(k, k^{\prime}\right) Z_{+}^{ \pm}\left(k^{\prime}\right)=\mathcal{S}_{+}^{\mathrm{o}}(k),
$$

where $k$ is real, the path $\Gamma^{\circ}$ lies in the real axis and is indented above $-k$,

$$
\begin{aligned}
& Z_{+}^{ \pm}(k)=H_{+}(k) \pm H_{-}(-k), \\
& \mathcal{S}_{+}^{o}(k)=\left[-V_{+}(k) \mp i S_{-}(-k)\right] K_{+}(k),
\end{aligned}
$$

and the complex kernel $\mathcal{K}^{\circ}$ is

$$
\mathcal{K}^{\mathrm{o}}\left(k, k^{\prime}\right)=\frac{1}{2 \pi} \frac{e^{2 i k^{\prime} b}}{k^{\prime}+k} \frac{K_{+}(k)}{K_{-}\left(k^{\prime}\right)} .
$$

Proof: We start with (4) of Proposition 1. For even kernel, we replace the independent variable $k$ by $-k$ in (4a) and change the integration variable from $k^{\prime}$ to $-k^{\prime}$ in (4). For odd kernel, we apply the same operations to these equations with the equation order reversed.

For even kernel (4) become

$$
\begin{gathered}
i V_{+}(-k)+\frac{H_{+}(-k)}{K_{-}(k)}+\int_{\Gamma^{\mathrm{e}}} \frac{d k^{\prime}}{2 \pi} \frac{e^{-2 i k^{\prime} b}}{k^{\prime}+k} \frac{H_{-}\left(k^{\prime}\right)}{K_{+}\left(k^{\prime}\right)}=0, \\
S_{-}(k)+\frac{H_{-}(k)}{K_{-}(k)}+\int_{\Gamma^{\mathrm{e}}} \frac{d k^{\prime}}{2 \pi} \frac{e^{-2 i k^{\prime} b}}{k^{\prime}+k} \frac{H_{+}\left(-k^{\prime}\right)}{K_{+}\left(k^{\prime}\right)}=0,
\end{gathered}
$$

where $k$ is now taken to be real. By adding and subtracting these equations, we derive (31)-(34), which concludes the proof for even kernel.

\footnotetext{
${ }^{3}$ The superscripts \pm here and in the remainder of the article are self-explanatory and should not be confused with the same symbols as subscripts, which denote analyticity in the upper or lower half plane.
} 
For odd kernel (4) become

$$
\begin{gathered}
V_{+}(k)+\frac{H_{+}(k)}{K_{+}(k)}-\int_{\Gamma^{\circ}} \frac{d k^{\prime}}{2 \pi} \frac{e^{2 i k^{\prime} b}}{k^{\prime}+k} \frac{H_{-}\left(-k^{\prime}\right)}{K_{-}\left(k^{\prime}\right)}=0, \\
i S_{-}(-k)+\frac{H_{-}(-k)}{K_{+}(k)}-\int_{\Gamma^{\circ}} \frac{d k^{\prime}}{2 \pi} \frac{e^{2 i k^{\prime} b}}{k^{\prime}+k} \frac{H_{+}\left(k^{\prime}\right)}{K_{-}\left(k^{\prime}\right)}=0,
\end{gathered}
$$

where $k$ is again taken to be real. By properly adding and subtracting these equations, we deduce (35)-(38), which completes the proof for odd kernel.

Clearly, the definitions of complex kernels for (31) and (35) are not unique. Another choice is

$$
\begin{aligned}
& \mathcal{K}^{\mathrm{e}}\left(k, k^{\prime}\right)=\frac{1}{2 \pi} \frac{e^{-2 i k^{\prime} b}}{k^{\prime}+k} \frac{K_{-}\left(k^{\prime}\right)}{K_{+}\left(k^{\prime}\right)}, \\
& \mathcal{K}^{\mathrm{o}}\left(k, k^{\prime}\right)=\frac{1}{2 \pi} \frac{e^{2 i k^{\prime} b}}{k^{\prime}+k} \frac{K_{+}\left(k^{\prime}\right)}{K_{-}\left(k^{\prime}\right)} .
\end{aligned}
$$

Formulas (41) and (42) are used for mathematical convenience in Lemma 1 below and in Section 3.2 in connection to an advection-diffusion problem. The corresponding functions entering (31) and (35) in place of (32), (33), (36), and (37) are

$$
\begin{aligned}
& Y_{-}^{ \pm}(k)=\frac{H_{+}(-k) \pm H_{-}(k)}{K_{-}(k)}, \\
& \mathcal{S}_{-}^{\mathrm{e}}(k)=-i V_{+}(-k) \mp S_{-}(k), \\
& Z_{+}^{ \pm}(k)=\frac{H_{+}(k) \pm H_{-}(-k)}{K_{+}(k)}, \\
& \mathcal{S}_{+}^{\mathrm{o}}(k)=-V_{+}(k) \mp i S_{-}(-k) .
\end{aligned}
$$

2.3.2. Complex kernel norm and iteration series in Fourier domain. Next, we outline the steps for solving (31) and (35) for algebraic $\tilde{K}(k)$ by invoking theory of 2nd-kind Fredholm equations [2, 23], which in turn relies on the definition of a norm for the complex kernels $\mathcal{K}^{\mathrm{e}, \mathrm{o}}$. Here, we discuss the case with a square integrable complex kernel [2] (finite " $L_{2}$-norm"). By allowing $k$ to move freely in the lower or upper half plane, the integration path in (31) or (35) can be deformed in the lower or upper half $k^{\prime}$-plane, respectively, to a contour $\Gamma_{<}$or $\Gamma_{>}$wrapped around appropriate branch cuts assigned to $\tilde{K}(k)$. Along these deformed contours, the kernels $\mathcal{K}^{\mathrm{e}, \mathrm{o}}\left(k, k^{\prime}\right)$ decay exponentially with $k^{\prime}$; see Section 3.2 for implementation of this idea to a symmetric kernel 
for advection-diffusion. Defining a trajectory $s(t)$ for $k$ and $k^{\prime}$ lying in $\Gamma_{<}$or $\Gamma_{>}$, so that $k=s(t)$ and $k^{\prime}=s\left(t^{\prime}\right)$ taking $-\infty<t, t<\infty$ for definiteness, (31) and (35) are recast to

$$
\mathcal{U}(k(t)) \pm \int_{-\infty}^{+\infty} d t^{\prime} \dot{s}\left(t^{\prime}\right) \mathcal{K}^{\mathrm{e}, \mathrm{o}}\left(s(t), s\left(t^{\prime}\right)\right) \mathcal{U}\left(s\left(t^{\prime}\right)\right)=\mathcal{S}(s(t))
$$

where $\dot{s}(t)=d s(t) / d t$ and $\mathcal{K}^{\mathrm{e}, \mathrm{o}}=O\left(e^{-2\left|\operatorname{Im} s\left(t^{\prime}\right)\right| b}\right)$ as $k^{\prime} \rightarrow \infty$.

We now state the following lemma, needed for Propositions 4 and 5 below:

LEMMA 1. For algebraic Fourier transform $\tilde{K}(k)$, analytic and invertible in the finite strip $D=\left\{k:-\xi_{0}<\operatorname{Im} k<\xi_{0}\right\}$, the $L_{2}-n o r m,\left\|\mathcal{K}^{\mathrm{e}, \mathrm{o}}\right\|$, defined by

$$
\left\|\mathcal{K}^{\mathrm{e}, \mathrm{o}}\right\|^{2} \equiv \int_{-\infty}^{+\infty} d t^{\prime} \int_{-\infty}^{+\infty} d t\left|\dot{s}\left(t^{\prime}\right) \dot{s}(t)\right|\left|\mathcal{K}^{\mathrm{e}, \mathrm{o}}\left(s(t), s\left(t^{\prime}\right)\right)\right|^{2}
$$

is finite $\left\|\mathcal{K}^{\mathrm{e}, \mathrm{o}}\right\|<\infty$.

Proof: The proof follows from (47) by use of alternative formulas (41) and (42) for the complex kernels. We partly symmetrize the complex kernel by the prescription of [2] and apply the definition of a norm for square integrable kernels $[2,23]$ so as to obtain (48). Integration in $t$ is performed first: Choosing the trajectory $s(t)$ so that $s(t)=O(|t|)$ as $|t| \rightarrow \infty$ and noting that, for algebraic kernels considered here,

$$
\left|\frac{K_{-}\left(k^{\prime}\right)}{K_{+}\left(k^{\prime}\right)}\right| \rightarrow 1 \quad k^{\prime} \rightarrow \infty, \quad k^{\prime}=s\left(t^{\prime}\right),
$$

the integral in $t$ is absolutely convergent. The remaining integral in $t^{\prime}$ is also convergent because of the exponential decay of the integrand noted below (47).

Invoking the theory of Fredholm equations [2, 23] we can routinely state conditions for solutions to (31) and (35), especially the Fredholm alternative [2] for square integrable complex kernels. In operator notation, (31) and (35) are recast to

$$
\mathcal{U} \pm \mathcal{K U}=\mathcal{S}
$$

where $\mathcal{U}=Y_{-}$or $Z_{+}, \mathcal{K}=\mathcal{K}^{\mathrm{e}}$ or $\mathcal{K}^{\mathrm{o}}$, and $\mathcal{S}=\mathcal{S}_{-}^{\mathrm{e}}$ or $\mathcal{S}_{+}^{\mathrm{o}}$ is the source function. The solution to (50) is obtained iteratively via the Neumann sum

$$
\mathcal{U}=\sum_{m=0}^{M}(\mp 1)^{m} \mathcal{K}^{m} \mathcal{S}
$$

which amounts to expansion (28) for $U(k)$. The operators $\mathcal{K}^{m}$ here are represented by the iterated kernels defined by [2] 


$$
\mathcal{K}_{m}\left(k, k^{\prime}\right) \equiv \int_{\Gamma} d k^{\prime \prime} \mathcal{K}_{m-1}\left(k, k^{\prime \prime}\right) \mathcal{K}_{1}\left(k^{\prime \prime}, k^{\prime}\right), \quad \mathcal{K}_{1} \equiv \mathcal{K}, \quad m=2,3, \ldots,
$$

so that $\mathcal{K}^{m} \mathcal{S}$ is represented by the function

$$
\left(\mathcal{K}^{m} \mathcal{S}\right)(k) \equiv \int_{\Gamma} d k^{\prime} \mathcal{K}_{m}\left(k, k^{\prime}\right) \mathcal{S}\left(k^{\prime}\right),
$$

where the path $\Gamma$ coincides with $\Gamma^{\mathrm{e}}$ or $\Gamma^{\mathrm{o}}$ specified in Proposition 3.

In particular, we state without proof the following propositions regarding (47).

Proposition 4 [2, 23]. Iteration series $(28)$ for $U(k)$, or alternatively $(51)$ as $M \rightarrow \infty$, converges in the complex Fourier domain uniformly with $k$ inside the strip $\left\{k:-\xi_{0}<\operatorname{Im} k<\xi_{0}\right\}$ if $\left|\lambda_{1}\right|>1$, where $\lambda_{1}=\lambda_{1}(\epsilon)$ is the eigenvalue of the (partly symmetrized) kernel $\sqrt{\dot{s}\left(t^{\prime}\right) \dot{s}(t)} \mathcal{K}\left(s(t), s\left(t^{\prime}\right)\right)$ that has the smallest magnitude, and $\mathcal{K}$ is defined by (34) or (38); alternatively, $\mathcal{K}$ can be defined by (41) or (42).

Proposition 5 [2, 23] [Sufficient condition for existence of solution and convergence of iteration series in Fourier domain]. If

$$
\|\mathcal{K}\|<1,
$$

the iteration series (28) for $U(k)$, or alternatively (51) as $M \rightarrow \infty$, converges in the complex Fourier domain uniformly with $k$ inside the strip $\left\{k:-\xi_{0}<\right.$ $\left.\operatorname{Im} k<\xi_{0}\right\}$.

Because $\lambda_{1}$ is not known a priori, Proposition 4 becomes useful for applications if suitable lower bounds are obtained for $\left|\lambda_{1}\right|[2,23]$. For example, the practically appealing Proposition 5 can result from the inequality [2] $\left|\lambda_{1}\right| \geq\|\mathcal{K}\|^{-1}$. Improved lower bounds for $\left|\lambda_{1}\right|$ involve iterated kernels [2].

Assuming $\xi_{0}$ is independent of the physical strip length, $2 b,\|\mathcal{K}\|$ is a monotonically decreasing function of $2 b$ and, thus, (54) is satisfied for $b$ $>B$, or $\epsilon<e^{-2 B \xi_{0}}$ where $B$ is a lower bound of $b$. Hence, the question arises whether $B \xi_{0}$ can be very small, or $\epsilon$ be quite close to unity for rapid convergence. Evidently, the answer depends on the behavior of $\|\mathcal{K}\|$ near $b=$ 0 ; see Section 3.2.

Once $Y_{-}^{ \pm}(k)$ or $Z_{+}^{ \pm}(k)$ of Proposition 3 are determined, the functions $H_{ \pm}(k)$ are obtained via (32) or (36),

$$
H_{ \pm}(k)=\frac{Y_{-}^{+}(\mp k) \pm Y_{-}^{-}(\mp k)}{2}, \quad \text { or } \quad H_{ \pm}(k)=\frac{Z_{+}^{+}( \pm k) \pm Z_{+}^{-}( \pm k)}{2},
$$

by which $u(x)$ is obtained in view of Corollary 1 . The inversion integral formula (20) is required to converge with the path $\Gamma$ lying inside the strip $\left\{k:-\xi_{0}<\operatorname{Im} k<\xi_{0}\right\}$. 


\section{Steady advection-diffusion near absorbing strip}

Next, we illustrate the analytical computations involved in and the efficiency of our method by focusing on a steady advection-diffusion problem [7], where the unknown function $u(x)$ of (1) is the flux to the absorbing strip boundary, $f(x)=1, b=-a=P>0$, and

$$
K(x)=\frac{1}{\pi} e^{x} K_{0}(|x|) \text {. }
$$

As shown below, this choice of source function and kernel causes fortuitous algebraic simplifications.

In this case, the kernel $K(x)$ behaves as

$$
K(x)=O\left(x^{-1 / 2}\right) \quad \text { as } \quad x \rightarrow+\infty, \quad K(x)=O\left(|x|^{-1 / 2} e^{2 x}\right) \quad \text { as } \quad x \rightarrow-\infty .
$$

Thus, $\eta=\zeta=1 / 2$ and $\xi_{0}=1$ in (21). First, we obtain $\tilde{K}(k), K_{ \pm}(k)$ and $F(k)$ by using (2), (8), and (13) [21],

$$
\begin{aligned}
\tilde{K}(k) & =\frac{1}{\sqrt{k} \sqrt{k+2 i}}, \quad K_{+}(k)=\frac{1}{\sqrt{k+2 i}}, \quad K_{-}(k)=\frac{1}{\sqrt{k}}, \\
F(k) & =2 \frac{\sin (k P)}{k},
\end{aligned}
$$

where, for definiteness, the branch cut for $\sqrt{k}$ coincides with the positive imaginary axis and the branch cut for $\sqrt{k+2 i}$ is the half line $\{k: \operatorname{Re} k=$ $0, \operatorname{Im} k<-2\}$. It follows from (23) that

$$
\epsilon=e^{-4 P} \text {. }
$$

Second, we calculate the functions $V_{+}(k)$ and $S_{-}(k)$, which enter (22), using (17) and (18) along with the Cauchy integral formula $[2,15]$ :

$$
V_{+}(k)=\int_{C_{1}} \frac{d k^{\prime}}{2 \pi i} \frac{\left[e^{-i k^{\prime} P} F\left(k^{\prime}\right)\right]_{-}}{K_{+}\left(k^{\prime}\right)} \frac{1}{k^{\prime}-k} \equiv V_{0+}(k)+\epsilon V_{1+}(k),
$$

where

$$
\begin{aligned}
V_{0+} & =\int_{C_{1}} \frac{d k^{\prime}}{2 \pi i} \frac{\sqrt{k^{\prime}+2 i}}{i k^{\prime}} \frac{1}{k^{\prime}-k}=\frac{\sqrt{k+2 i}-\sqrt{2 i}}{i k}, \\
V_{1+} & =-\int_{C_{1}} \frac{d k^{\prime}}{2 \pi i} \sqrt{k^{\prime}+2 i} \frac{e^{-i 2 P\left(k^{\prime}+2 i\right)}}{i k^{\prime}} \frac{1}{k^{\prime}-k} \\
& =\frac{e^{i \pi / 4}}{i k} e^{4 P}\left[\sqrt{2} \operatorname{erfc}(\sqrt{4 P})-e^{-i \pi / 4} \sqrt{k+2 i} e^{-i 2 P k} \operatorname{erfc}(\sqrt{2 P(2-i k)})\right],
\end{aligned}
$$


and

$$
S_{-}(k) \equiv S_{0-}=\int_{C_{2}} \frac{d k^{\prime}}{2 \pi i} \frac{1-e^{2 i P k^{\prime}}}{i \sqrt{k^{\prime}}} \frac{1}{k^{\prime}-k}=\frac{i}{\sqrt{k}}\left[1-e^{i 2 P k} \operatorname{erfc}(\sqrt{i 2 P k})\right],
$$

where the contours $C_{1}$ and $C_{2}$ are defined by (4) taking $\alpha=-2$ and $\beta=0$, and $\operatorname{erfc}(z)=\frac{2}{\sqrt{\pi}} \int_{z}^{\infty} d t e^{-t^{2}}$ is the complementary error function. We note that, for definiteness, $-2<c_{1}<0$ in (62) and (63) since the split integrands acquire a simple pole at $k^{\prime}=0$. In inversion formula (20) and its iteration counterpart, (28d), the contour $\Gamma$ must lie inside the complex strip $\{k:-2<\operatorname{Im} k<0\}$.

\subsection{Iteration series via integral-equation pair}

In this section, we apply Proposition 2, specifically the recursive formulas (26), to find an approximate solution to the given advection-diffusion problem. We show that our formulation not only reproduces the high-Péclet asymptotic expansion of [7] but also enables the derivation of useful lower bounds for convergence via Proposition 5.

3.1.1. Zeroth-order approximation. First, we use the recursive formulas (26) for $n=0$. The zeroth-order terms for the unknowns $H_{ \pm}$read

$$
\begin{aligned}
& H_{0+}(k)=-\frac{1}{\sqrt{k+2 i}} \frac{\sqrt{k+2 i}-\sqrt{2 i}}{i k}, \\
& H_{0-}(k)=K_{-}(k)\left[-S_{-}(k)+\int_{C_{2}} \frac{d k_{0}}{2 \pi i} \frac{e^{i 2 k_{0} P} H_{0+}\left(k_{0}\right)}{K_{-}\left(k_{0}\right)} \frac{1}{k_{0}-k}\right],
\end{aligned}
$$

by which, in view of (28b), we obtain

$$
\begin{aligned}
U(k) & \sim U_{0}=\frac{1}{K_{+} K_{-}}\left[e^{i k P} H_{0+}+e^{-i k P} H_{0-}+F\right] \\
& =e^{i k P} \frac{\sqrt{2 i}}{i \sqrt{k}}+e^{-i k P} \sqrt{k+2 i} \int_{C_{2}} \frac{d k_{0}}{2 \pi i} \frac{e^{i 2 k_{0} P}}{i \sqrt{k_{0}} \sqrt{k_{0}+2 i}} \frac{\sqrt{2 i}}{k_{0}-k} .
\end{aligned}
$$

Inversion of the last formula by (28d) is carried out by wrapping the contours in the $k$ - and $k_{0}$-planes around the branch cuts of $\sqrt{k+2 i}$ and $\sqrt{k_{0}}$, and changing integration variables by $i(P-x)(k+2 i) \equiv \tau^{2}$ and $-i k_{0} \equiv y_{0}^{2}$, respectively.

Thus, we obtain the following result.

RESUlt 1. The zeroth-order solution, $u_{0}(x)$, for the advection-diffusion problem is 


$$
\begin{aligned}
u(P \chi) \sim u_{0}(P \chi)= & \sqrt{\frac{2}{\pi P}} \frac{1}{\sqrt{1+\chi}}+\frac{1}{\pi^{2}} \sqrt{\frac{2}{P}} \frac{1}{\sqrt{1-\chi}} \int_{-\infty}^{+\infty} d \tau \tau^{2} e^{-\tau^{2}} \\
& \times \int_{-\infty}^{+\infty} d y_{0} \frac{e^{-2 P y_{0}^{2}}}{\tau^{2}+P(1-\chi)\left(2+y_{0}^{2}\right)} \frac{1}{\sqrt{2+y_{0}^{2}}}
\end{aligned}
$$

where $\chi=x / P,-1<\chi<1$.

The zeroth-order iteration yields both end point singularities of $u(x)$, at $\chi=$ \pm 1 . The variable $\chi$ was introduced for direct comparisons with the results in Section 4 of [7]. The integral of (67) can be simplified [7], and $u_{0}(x)$ becomes

$$
\begin{aligned}
u_{0}(P \chi)= & \sqrt{\frac{2}{\pi P}}\left[\frac{1}{\sqrt{1+\chi}}-\frac{1}{\sqrt{\pi}}\right. \\
& \left.\times \int_{-\infty}^{+\infty} d \xi e^{-(1+\chi) \xi^{2}} \operatorname{erfc}\left(\sqrt{\left(2 P+\xi^{2}\right)(1-\chi)}\right)+\frac{e^{2 P \chi} K_{0}(2 P)}{\pi \sqrt{1-\chi}}\right],
\end{aligned}
$$

which is also given by equation (4.15) in [7].

3.1.2. First-order approximation. It is of interest to carry out the iteration (26) to one more order, taking $n=1$, before we describe the results for arbitrary $n$ in Section 3.1.3. Thus, we have

$$
\begin{aligned}
H_{1+}(k)= & -K_{+}(k) V_{1+}(k)-K_{+}(k) \int_{C_{1}} \frac{d k_{0}}{2 \pi i} \frac{e^{-i 2\left(k_{0}+2 i\right) P} H_{0-}\left(k_{0}\right)}{K_{+}\left(k_{0}\right)} \frac{1}{k_{0}-k} \\
= & -\frac{\sqrt{2 i}}{\sqrt{k+2 i}} \int_{C_{1}} \frac{d k_{0}}{2 \pi i} \frac{e^{-i 2\left(k_{0}+2 i\right) P} \sqrt{k_{0}+2 i}}{\sqrt{k_{0}}} \frac{1}{k_{0}-k} \\
& \times \int_{C_{2}} \frac{d k_{1}}{2 \pi i} \frac{e^{i 2 k_{1} P}}{i \sqrt{k_{1}} \sqrt{k_{1}+2 i}} \frac{1}{k_{1}-k_{0}}, \\
H_{1-}(k)= & K_{-}(k) \int_{C_{2}} \frac{d k_{0}}{2 \pi i} \frac{e^{i 2 k_{0} P} H_{1+}\left(k_{0}\right)}{K_{-}\left(k_{0}\right)} \frac{1}{k_{0}-k},
\end{aligned}
$$

by which $U_{1}(k)$ is obtained via (28c), 


$$
\begin{aligned}
U_{1}(k)= & \frac{1}{K_{+} K_{-}}\left[e^{i k P} H_{1+}+e^{-i k P} H_{1-}\right] \\
= & -\sqrt{2 i k} e^{i k P} \int_{C_{1}} \frac{d k_{0}}{2 \pi i} \frac{e^{-i 2\left(k_{0}+2 i\right) P} \sqrt{k_{0}+2 i}}{\sqrt{k_{0}}} \frac{1}{k_{0}-k} \int_{C_{2}} \frac{d k_{1}}{2 \pi i} \\
& \times \frac{e^{i 2 k_{1} P}}{i \sqrt{k_{1}} \sqrt{k_{1}+2 i}} \frac{1}{k_{1}-k_{0}}-\sqrt{2 i(k+2 i)} e^{-i k P} \int_{C_{2}} \frac{d k_{0}}{2 \pi i} \frac{e^{i 2 k_{0} P} \sqrt{k_{0}}}{\sqrt{k_{0}+2 i}} \\
& \times \frac{1}{k_{0}-k} \int_{C_{1}} \frac{d k_{1}}{2 \pi i} \frac{e^{-i 2\left(k_{1}+2 i\right) P} \sqrt{k_{1}+2 i}}{\sqrt{k_{1}}} \frac{1}{k_{1}-k_{0}} \int_{C_{2}} \frac{d k_{2}}{2 \pi i} \\
& \times \frac{e^{i 2 k_{2} P}}{i \sqrt{k_{2}} \sqrt{k_{2}+2 i}} \frac{1}{k_{2}-k_{1}} .
\end{aligned}
$$

Inversion of this formula is carried out by (28d) via deforming the integration paths around the branch cuts in the upper or lower halves of the $k$-and $k_{j}$-planes $(j=0,1,2)$, following the integration procedure of Section 3.1.1.

After some algebra, we obtain the following result.

RESULT 2. The first-order solution, $u_{1}(x)$, of the advection-diffusion problem is

$$
\begin{aligned}
& u_{1}(P \chi) \\
& =\sqrt{\frac{2}{P}} \frac{1}{\pi^{3} \sqrt{1+\chi}} \int d \tau \tau^{2} e^{-\tau^{2}} \int d y_{0} \frac{e^{-2 P y_{0}^{2}}}{\tau^{2}+P(1+\chi)\left(2+y_{0}^{2}\right)} \frac{y_{0}^{2}}{\sqrt{2+y_{0}^{2}}} \\
& \quad \times \int d y_{1} \frac{e^{-2 P y_{1}^{2}}}{2+y_{0}^{2}+y_{1}^{2}} \frac{1}{\sqrt{2+y_{1}^{2}}}+\sqrt{\frac{2}{P} \frac{1}{\pi^{4} \sqrt{1-\chi}} e^{-2 P(1-\chi)} \int d \tau \tau^{2} e^{-\tau^{2}}} \\
& \quad \times \int d y_{0} \frac{e^{-2 P y_{0}^{2}}}{\tau^{2}+P(1-\chi)\left(2+y_{0}^{2}\right)} \frac{y_{0}^{2}}{\sqrt{2+y_{0}^{2}}} \int d y_{1} \frac{e^{-2 P y_{1}^{2}}}{y_{1}^{2}+y_{0}^{2}+2} \frac{y_{1}^{2}}{\sqrt{2+y_{1}^{2}}} \\
& \quad \times \int d y_{2} \frac{e^{-2 P y_{2}^{2}}}{y_{2}^{2}+y_{1}^{2}+2} \frac{1}{\sqrt{2+y_{2}^{2}}}, \quad-1<\chi<1 .
\end{aligned}
$$

The symbol $\int$ here and in the remainder of this article denotes integration from $-\infty$ to $+\infty$ unless it is stated otherwise. Again, the iteration yields both end point singularities of $u(x)$.

3.1.3. $n$ th-order approximation $(n \geq 1)$. The iteration procedure of $(26)$ can be repeated to arbitrary order. For $n=0$, the solution is given by (67). By induction for $n \geq 1$, the integral formula for arbitrary $n$ is obtained. 
RESULT 3 [nth-order coefficient of iteration series for $u(x)$ ]. The iteration series $u_{0}(x)+u_{1}(x)+\cdots+u_{n}(x)+\cdots$ for the flux on the physical strip boundary has the general coefficient

$u_{n}(P \chi)=\frac{1}{\pi^{2}} \sqrt{\frac{2}{P}}\left[\frac{1}{\sqrt{1+\chi}} I_{2 n}(1+\chi)+\frac{e^{-2(1-\chi) P}}{\sqrt{1-\chi}} I_{2 n+1}(1-\chi)\right], \quad n \geq 1$,

where $\chi=x / P,-1<\chi<1$,

$$
\begin{aligned}
I_{m}(v)= & \int d \tau \tau^{2} e^{-\tau^{2}} \int d y_{0} \frac{Q_{0}}{P v\left(2+y_{0}^{2}\right)+\tau^{2}} \int d y_{1}\left(R_{0} Q_{1}\right) \int d y_{2}\left(R_{1} Q_{2}\right) \cdots \\
& \times \int d y_{m-1}\left(R_{m-2} Q_{m-1}\right), \quad m \geq 2 \\
R_{m}= & \frac{y_{m}^{2}}{\pi\left(y_{m}^{2}+y_{m+1}^{2}+2\right)}, \quad Q_{m}=\frac{e^{-2 P y_{m}^{2}}}{\sqrt{1+y_{m}^{2}}} .
\end{aligned}
$$

Each iteration accounts for both singularities of $u(x)$, which are the sole singularities allowed by the present, logarithmically singular (as $x \rightarrow 0$ ) kernel $K(x)[13,24]$. Equation (73) is in agreement with equations (4.21) in [7], which were derived via iterations of $(1)$ in the $x$-coordinate space. ${ }^{4,5}$ It has not been possible to evaluate the integrals $I_{m}$ of (74) in simple closed form for arbitrary $m$, but the numerical integrations can be carried out efficiently by using recursion [7]. In Section 3.2, we study the convergence of $\sum_{n=0}^{\infty} U_{n}(k)$ for advection-diffusion by invoking Proposition 5 of Section 2.3.2.

\subsection{Convergence by complex-kernel norm}

Next, we address the issue of convergence of scheme (28), which properly leads to the Neumann series (51) of Section 2.3.2. First, we symmetrize $K(x)$ fully by defining the modified kernel $K_{\text {mod }}=e^{-x} K(x)=(1 / \pi) K_{0}(|x|)$; thus, $\tilde{K}_{\text {mod }}(k)=\left(k^{2}+1\right)^{-1 / 2}$, which is a function analytic and invertible inside the complex strip $D=\{k:-1<\operatorname{Im} k<1\}$. The unknown and source functions are transformed to $u_{\text {mod }}(x)=e^{-x} u(x)$ and $f_{\text {mod }}(x)=e^{-x} f(x)=e^{-x}$. Hence, by alternative formula (41) the complex kernel $\mathcal{K}_{\text {mod }}^{\mathrm{e}}\left(k, k^{\prime}\right)$ is

$$
\mathcal{K}_{\text {mod }}^{\mathrm{e}}\left(k, k^{\prime}\right)=\frac{1}{2 \pi} \frac{e^{-2 i k^{\prime} P}}{k^{\prime}+k} \sqrt{\frac{k^{\prime}+i}{k^{\prime}-i}} .
$$

The upper half plane is $D_{\mathrm{u}}=\{\operatorname{Im} k>-1\}$ and the lower half plane is $D_{1}=$ $\{\operatorname{Im} k<1\}$.

\footnotetext{
${ }^{4}$ Equation (73) agrees with the statement by equation (1.5) in [13], which results from Riesz's theorem for bounded linear functionals.

${ }^{5}$ We alert the reader that our notation in (73)-(75) differs from the notation in [7].
} 
Second, by resorting to Proposition 3 we expose the exponential decay of $\mathcal{K}_{\text {mod }}^{\mathrm{e}}\left(k, k^{\prime}\right)$ by deforming the integration path for the integral of (31) in the lower half $k^{\prime}$-plane, where the contour is wrapped around the branch cut for $\sqrt{k^{\prime}+i}$. Using (48) with the trajectory $s(t)=-i-i t^{2}$, we obtain, after some algebra,

$$
\begin{aligned}
\left\|\mathcal{K}_{\text {mod }}^{\mathrm{e}}\right\|^{2} & =\frac{4 e^{-4 P}}{(2 \pi)^{2}} \int d t \int d t^{\prime}\left|t t^{\prime}\right| \frac{e^{-4 P t^{\prime} 2}}{\left(t^{2}+t^{\prime 2}+2\right)^{2}} \frac{t^{\prime 2}}{t^{\prime 2}+2} \\
& =\frac{e^{-4 P}}{\pi^{2}}\left[(1+8 P) e^{8 P} E_{1}(8 P)-1\right],
\end{aligned}
$$

where $E_{1}(z)$ is the exponential integral [25],

$$
E_{1}(z)=\int_{z}^{\infty} d t \frac{e^{-t}}{t} .
$$

Proposition 5 combined with (77) entails the following result.

RESULT 4 (Lower bound for $P$ ). The iteration series $U_{0}(k)+$ $U_{1}(k)+\cdots+U_{n}(k)+\cdots$ for the Fourier transform of $u(x)$, defined in the complex strip $D=\{k:-1<\operatorname{Im} k<1\}$, converges uniformly in D provided that

$$
(1+8 P) e^{4 P} E_{1}(8 P)-e^{-4 P}<\pi^{2},
$$

where the left-hand side is a monotonically decreasing function of $P$. The numerical solution of (79) yields [26]

$$
P>1.3356 \times 10^{-6} \text {. }
$$

This result reveals an astonishingly small lower bound for $P$, which comes from the monotonicity of $\left\|\mathcal{K}_{\text {mod }}\right\|$ with $P$ and the logarithmic singularity with a small prefactor of this norm as $P \rightarrow 0,{ }^{6}$

$$
\left\|K_{\text {mod }}^{\mathrm{e}}\right\|^{2} \sim \frac{1}{\pi^{2}}\left(\ln \frac{1}{8 P}-\gamma-1\right) .
$$

Indeed, use of (81) in Proposition 5 yields

$$
P>\frac{e^{-\pi^{2}-1-\gamma}}{8},
$$

which agrees well with $(80) ; \gamma$ is Euler's constant. An explicit calculation using (81) shows that $\|\mathcal{K}\|$ has sufficiently small values to guarantee rapid convergence of $\sum_{n} U_{n}(k ; \epsilon)$ even when $P=O\left(10^{-4}\right)$.

We conclude that condition (54) and its product, (79), effectively explain the remarkable overlap of the approximations used in [7] for high and low $P$; see Section 4 for a discussion of this intimate relation between convergence and behavior with $b \xi_{0}$ of $\|\mathcal{K}\|$ for algebraic $\tilde{K}(k)$.

${ }^{6}$ We use $E_{1}(z) \sim-\ln z-\gamma$, where $|z| \ll 1$ and $|\operatorname{Arg} z|<\pi$. 
(a)

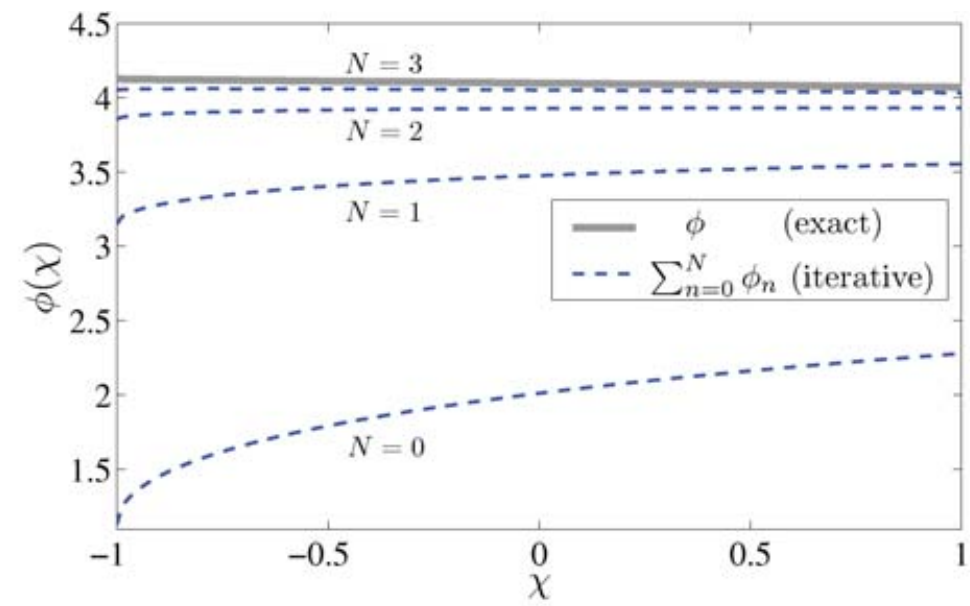

(b)

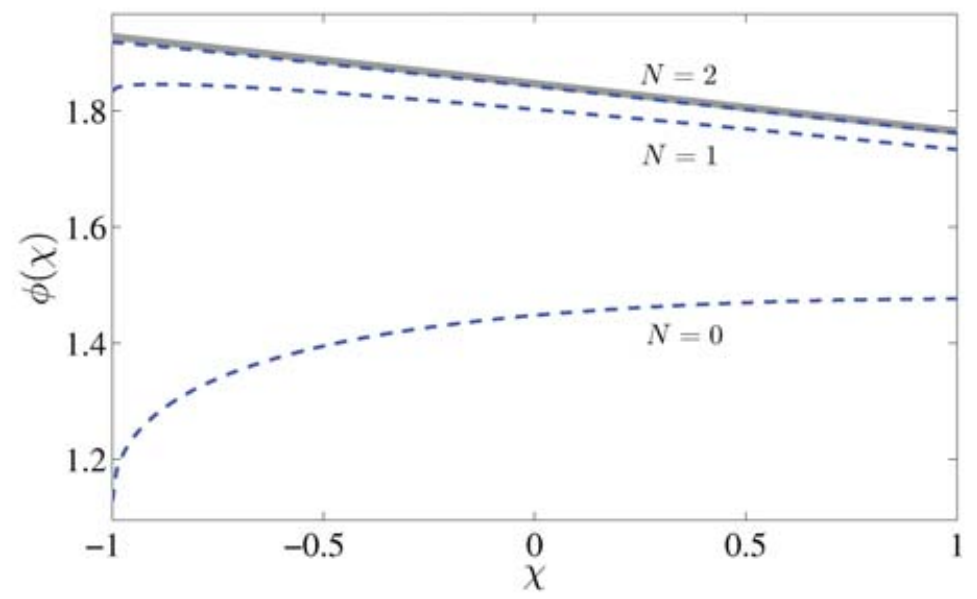

(c)

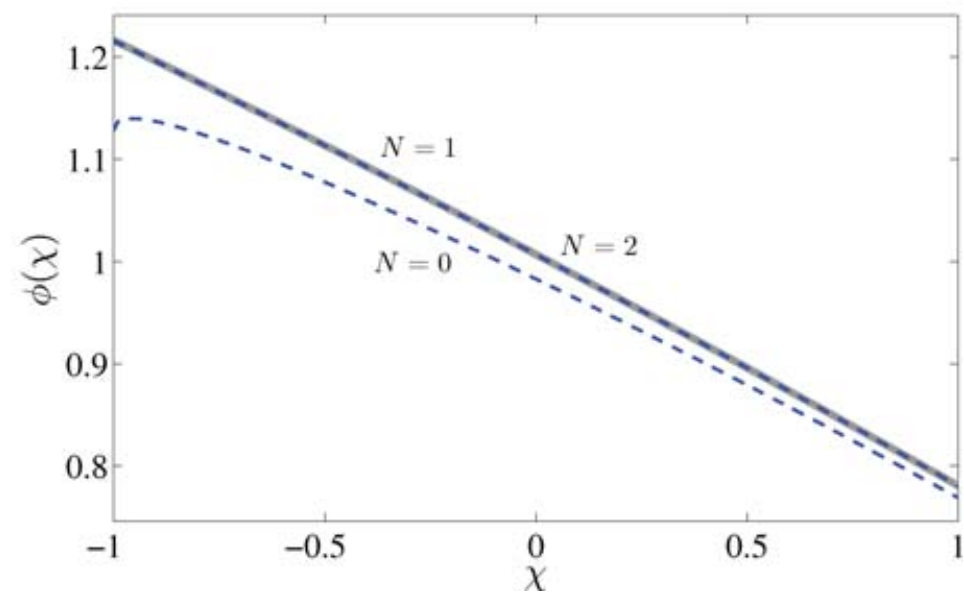

Figure 1. Numerical solution for $\phi(\chi)$ compared with our iteration series (28): (a) $P=10^{-3}$ and iteration number $N=0,1,2,3$, (b) $P=10^{-2}$ and $N=0,1,2$, and (c) $P=10^{-1}$ and $N=$ $0,1,2$. 
(a)

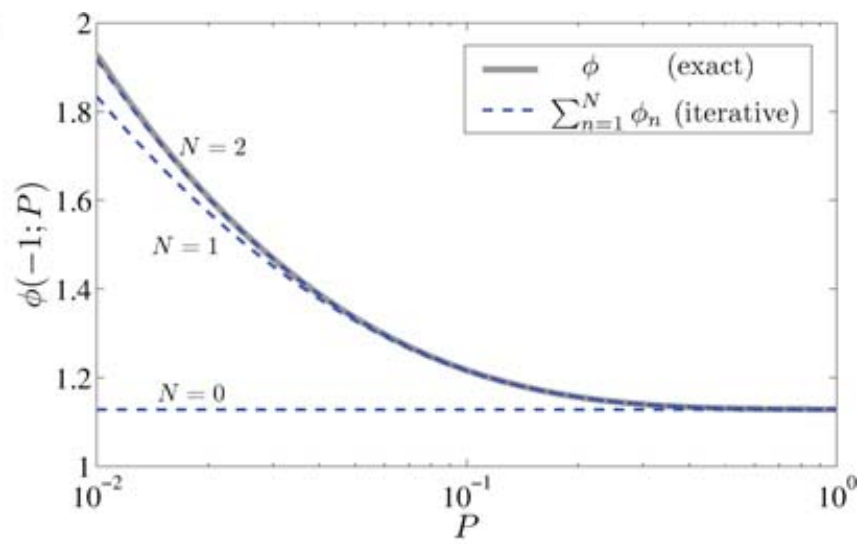

(b)

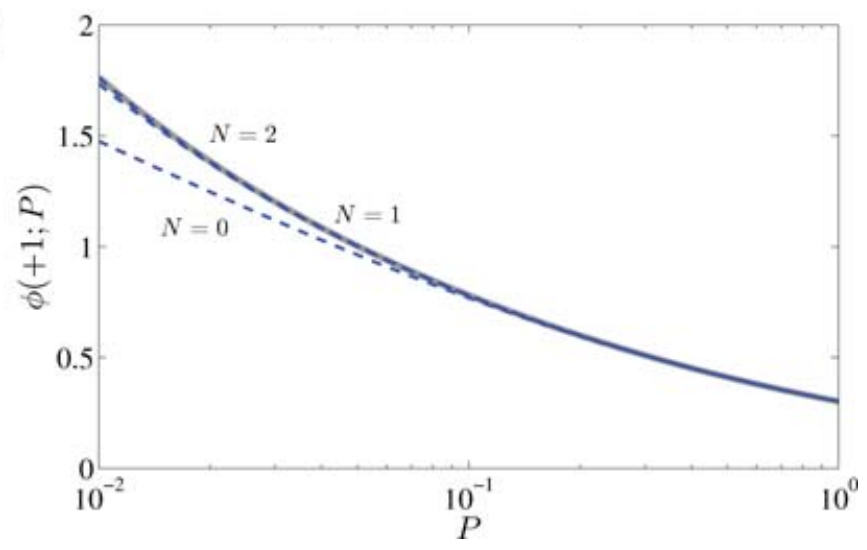

Figure 2. Numerical solution and iteration series with $N=0,1,2$ for $\phi(\chi ; P)$ at the strip end points, $\chi=-1$ and 1 , as functions of $P$ : (a) $\chi=-1$ and (b) $\chi=1$.

\subsection{Graphical representations}

Next, we provide comparisons for different values of $\epsilon$ (i.e., $P$ ) and $\chi=$ $x / P(-1<\chi<1)$ of the series (28) with the numerical solution of the BVP corresponding to (1). The details of the numerical method are described elsewhere [7]. For convenience in our comparisons, we define and use the continuous functions

$$
\phi(\chi)=\phi(\chi ; P)=\sqrt{P\left(1-\chi^{2}\right)} u(P \chi), \quad \phi_{n}(\chi)=\sqrt{P\left(1-\chi^{2}\right)} u_{n}(P \chi) .
$$

In Figure 1, we show the numerical solution for $\phi(\chi ; P)$ and the corresponding series $\sum_{n=0}^{N} \phi_{n}(\chi)$ as functions of $\chi$ for different values of the parameter $P$ and iteration number $N: P=10^{-3}$ and $N=0,1,2,3$ in Figure $1(\mathrm{a}), P=10^{-2}$ and $N=0,1,2$ in Figure $1(\mathrm{~b})$, and $P=10^{-1}$ and $N=0,1,2$ in Figure 1(c), where the maximum value of $N$ in each case is chosen for sufficient accuracy. In Figure 2, we show the numerical solution and the iteration series for $\phi(\chi ; P)$ evaluated at the strip endpoints, $\chi=-1$ [Figure 2(a)] and $\chi=1$ 
[Figure 2(b)] as functions of $P$ for different values of $N$. Similar comparisons have been shown in [7] for a circular physical boundary, which can be mapped to the finite strip via a simple conformal mapping [7].

\section{Conclusions}

In this article, we solved iteratively the first-kind integral equation (1) with kernel that decays exponentially; $K(x)$ has asymptotic limits (21) and algebraic Fourier transform that is analytic and invertible in a finite complex strip. As a starting point and without imposition of kernel symmetry, we considered the case where (1) stems from a well-posed BVP and thus has a unique integrable solution $u(x)$. Using the Wiener-Hopf technique, we converted (1) to a pair of coupled integral relations for Fourier transforms in the complex plane. Accordingly, we formulated the iteration method (26) via treating as small the coupling constant $\epsilon$, Equation (23), of these integral relations. By our prescription, $-\ln \epsilon$ admits a simple interpretation: It is the product of two strip lengths, namely, $b-a$, the length of the physical strip where boundary conditions are given in the BVP, and $2 \xi_{0}$, the width of the strip of analyticity of the kernel Fourier transform, where its inversion is carried out consistently with the asymptotic behavior of the kernel in the physical space. Our approximations produce all end point singularities of the actual solution at each iteration.

For difference kernels with odd or even symmetry, a unique, square integrable solution, $u(x)$, of (1) exists on the basis of the Hilbert-Schmidt theorem [2] when the kernel $K(x)$ describes a Sturm-Liouville system [2, 23]. We extend the analysis to singular, nonsquare integrable $u(x)$ by reducing the pair of integral relations for (1) to 2nd-kind Fredholm equations with kernels, $\mathcal{K}\left(k, k^{\prime}\right)$, of two complex variables. These equations are solved subject to the finiteness of the properly defined kernel norm in the complex $k, k^{\prime}$-space. In particular, the iteration series for the unknown Fourier transforms are identified with suitable Neumann series subject to established convergence criteria. By virtue of Propositions 4 and 5, we conclude that the rapidity of convergence of the iteration series does not depend exclusively on the magnitude of the coupling parameter, $\epsilon$. A complete characterization of the iteration series requires knowing the value of the complex-kernel norm, $\|\mathcal{K}\|$, defined by (48) in Lemma 1.

To validate the iteration method and exemplify the role of $\|\mathcal{K}\|$ we compared the iteration series with the numerical solution of a classic BVP describing steady advection-diffusion [7]. This problem involves a positive parameter, $P$, the Péclet number, so that in nondimensional variables the length of the physical strip is $b-a=2 P$ and the coupling constant is $\epsilon=e^{-4 P}$. Our computations showed that only 3 or 4 terms of the iteration series suffice for very good accuracy even when $P$ is small, $P=O\left(10^{-3}\right)$ and $\epsilon$ is quite close to unity. This property is understood by noticing that $\|\mathcal{K}\|$, interpreted as an $L_{2}$-norm along 
the appropriate path of the complex plane, is monotonically decreasing with $P$ and is logarithmically singular at $P=0$ with relatively small, constant prefactor.

A few general remarks are in order about $\|\mathcal{K}\|$, defined in the $L_{2}$-sense, when $\tilde{K}(k)$ is an algebraic function, even or odd, analytic and invertible in a symmetric, finite strip of fixed width $2 \xi_{0}$ in the complex plane. With

$$
\frac{K_{+}(k)}{K_{-}(k)}= \pm 1 \quad \text { as } \quad|k| \rightarrow \infty,
$$

it follows from (41) or (42) that

$$
\mathcal{K}\left(k, k^{\prime}\right) \sim \pm \frac{1}{2 \pi} \frac{e^{\mp 2 i b k^{\prime}}}{k+k^{\prime}} \quad \text { as } \quad k^{\prime} \rightarrow \infty,
$$

where the sign in front depends on the branch of $\tilde{K}(k)$ by (84) and the sign in the exponent depends on the kernel symmetry. Consequently, by (85) the integral (48) for $\|\mathcal{K}\|$, being monotonically decreasing with $2 b$ (the length of the physical strip), becomes logarithmically singular as $4 b \xi_{0} \rightarrow 0$ apparently regardless of the nature of singularities in $x$ of the kernel $K(x)$. We are tempted to expect that a lower bound for the product $4 b \xi_{0}$ such that the iteration series converges in the $k$-plane, although dependent on the precise expansion for $\|\mathcal{K}\|$ as $4 b \xi_{0} \rightarrow 0$, can in principle be small.

The analysis presented in this article unifies and generalizes the formulations in [3, 14], and elucidates results in [7] by showing an intimate connection of asymptotic analysis for high Péclet numbers to aspects of 2nd-kind Fredholm equations in the Fourier domain. There are open questions left for future work. From the viewpoint of applications, one should identify and solve by this iteration method problems where the kernel $K(x)$ has nonlogarithmic singularities; this task is the subject of a work in progress. The analytical framework developed here will hopefully become a common tool in software for solving first-kind integral equations and associated linear, mixed BVPs.

\section{References}

1. I. STAKGOLD, Green's Functions and Boundary-Value Problems, 2nd ed., Wiley-Interscience, New York, 1998.

2. M. Masujima, Applied Mathematical Methods in Theoretical Physics, Wiley-VCH, Weinheim, Germany, 2005.

3. B. NoBle, Methods Based on the Wiener-Hopf Technique for the Solution of Partial Differential Equations, 2nd ed., Chap. V, Chelsea, New York, 1988.

4. N. I. Muskhelishvili, Singular Integral Equations: Boundary Problems of Function Theory and Their Applications to Mathematical Physics, Dover, New York, 1992.

5. L. VAN WiJngaARden, Asymptotic solution of a diffusion problem with mixed boundary conditions, Proc. Koninkl. Nederl. Akad. Wet. 69:263-276 (1966).

6. V. A. MAKsimov, On the determination of the shape of bodies formed by solidification of the fluid phase of the stream, J. Appl. Math. Mech. (Prikl. Mat. Mekh.) 40:264-272 (1976). 
7. J. Choi, D. Margetis, M. Z. Bazant, and T. M. Squires, Steady advection-diffusion around finite absorbers in two-dimensional potential flows, J. Fluid Mech. 536:155-184 (2005).

8. V. A. Chugunov and K. G. Kornev, Dynamics of ice-rock barriers under conditions of freezing of filtering rocks, J. Eng. Phys. (Inzh.-Fiz. Zh.) 51:981-986 (1986).

9. K. G. Kornev and V. A. Chugunov, Determination of the equilibrium shape of the bodies formed during the solidification of filtration flow, J. Appl. Math. Mech. (Prikl. Mat. Mekh.) 52:773-778 (1988).

10. K. Kornev and G. Mukhamadullina, Mathematical theory of freezing for flow in porous media, Proc. Roy. Soc. London A 447:281-297 (1994).

11. M. M. Alimov, K. G. Kornev, and G. L. Mukhamadullina, The equilibrium shape of an ice-soil body formed by liquid flow past a pair of freezing columns, J. Appl. Math. Mech. (Prikl. Mat. Mekh.) 58:873-888 (1994).

12. M. Alimov, K. Kornev, and G. Mukhamadullina, Hysteretic effects in the problems of artificial freezing, SIAM J. Appl. Math. 59:387-410 (1998).

13. V. M. AleKsandrov, Asymptotic methods in the mechanics of continuous media: Problems with mixed boundary conditions, J. Appl. Math. Mech. (Prikl. Math. Mekh.) 57:321-327 (1993).

14. A. D. Polyanin and A. V. Manzhirov, Handbook of Integral Equations, pp. 521-524, CRC Press, New York, 1998.

15. G. Carrier, M. Krook, and C. E. Pearson, Functions of a Complex Variable, Chap. 8, Hod Books, New York, 1983.

16. G. E. Latta, The solution of a class of integral equations, Rat. Mech. Anal. 5:821-831 (1956).

17. J. M. Myers, Wave scattering and the geometry of a strip, J. Math. Phys. 6:1839-1846 (1965); Derivation of a matrix Painlevé equation germane to wave scattering by a broken corner, Physica D 11:51-89 (1984).

18. V. L. RVACHEV, The pressure on an elastic half-space of a stamp in the form of a strip in a plane, Prikl. Mat. Mekh. 20:163-179 (1956) (In Russian).

19. C. D. Green, Integral Equation Methods, Chap. 4, Barnes \& Noble, New York, 1969.

20. D. S. Jones, Diffraction by a waveguide of finite length, Proc. Camb. Phil. Soc. 48:118-134 (1952).

21. Bateman Manuscript Project, Higher Transcendental Functions, Vol. II, edited by A. Erdélyi, Chap. VII, Krieger, Malabar, Florida, 1981.

22. M. G. KREIN, Integral equations on a half line with kernel depending upon the difference of the arguments, Am. Math. Transl. 22:163-288 (1962).

23. F. G. Tricomi, Integral Equations, Chap. II, Dover, New York, 1957.

24. T. CARLEMAN, Über die Abelsche Integralgleichung mit konstanten Integrationsgrenzen, Math. Zeitschrift 15:111-120 (1922); C. E. PEARson, On the finite strip problem, Quart. Appl. Math. XV:203-208 (1957).

25. W. Gautschi and W. F. Cahill, Exponential integral and related functions, Chap. 5 in Handbook of Mathematical Functions (M. Abramowitz and I. A. Stegun, Eds.), Dover, New York, 1972.

26. S. Wolfram, The Mathematica Book, p. 744, Cambridge University Press, New York, 1991.

Massachusetts Institute of TeChNOLOGy

(Received June 29, 2005) 